\title{
Ethical Dilemma of Error Disclosure in Wrong-Site Craniotomy: A Systematic Review
}

\author{
Taiwo Akhigbe ${ }^{1,2 *}$, Ardalan Zolnourian ${ }^{1}$ \\ ${ }^{1}$ Department of Neurosurgery, University Hospital Southampton, Southampton, UK \\ 2 Altnalgelvin Area Hospital, Northern Ireland, UK
}

Corresponding Author: Taiwo Akhigbe, MBBS, MCh, MSc, PgD, Department of Neurosurgery, University Hospital Southampton, Tremona Rd, Southampton SO16 6YD, UK. E-mail: akhigbetaiwo@yahoo.com

Received January 28, 2017; Accepted March 17, 2017; Online Published March 28, 2017

\begin{abstract}
Introduction: Disclosure of errors to patients has long been fully endorsed by professional organizations and bioethicists because of its respect for patient autonomy, enhances informed decision-making and upholds the physician decision to tell the truth. Wrong site craniotomy have massive effects on both the patients and the surgeons. The purpose of this review was to apply moral philosophical analysis to this delicate and important issue that will for sure continually confront neurosurgeons in practice.

Methods: A comprehensive selected literature search was performed to establish evidence for informed and evidenced based discussion including the current literature relating to wrong-site craniotomy error disclosure. A total of 232 articles were gathered, but after removing duplicates, editorial comments and correspondences, only two articles met the criteria.

Results: In terms of the quality of the gathered evidence, only peer reviewed papers reached the limit. Generally, there is paucity of literature in the ethics of wrong site craniotomy. As a result, only two articles met the criteria (Cohen and Wu et al.).

Conclusion: Deontology strongly believed that all major accidental errors which occur when facing patients should be disclosed.

This is due to the fact that surgeons are duty-bound to do the right thing including not lying, respecting patients' dignity, practicing beneficence, sympathy and acting with gratitude and conscience without arrogance.

Keywords: Deontology; Truth Disclosure; Ethical Dilemma; Craniotomy

Citation: Akhigbe T, Zolnourian A. Ethical dilemma of error disclosure in wrong-site craniotomy: a systematic review. Int J Med Rev. 2017;4(1):17-21. doi: 10.29252/ijmr-040104.
\end{abstract}

\section{Introduction}

Medical errors are the eight leading causes of death in the United States accounting for annual patient fatalities that will equivalently result in three jumbo jets crashing every two days. ${ }^{1}$ Error disclosure is very fundamental to error prevention. Clinicians face a dilemma when deciding on whether and how harmful medical or surgical errors were for patients. This is issue is because of their fear of lawsuits and self-perception of incompetence and unskillfulness which could be dispelled by organizational cultures emphasizing safety rather than blame. Harvard Medical Practice found out that more than $70 \%$ of errors resulting in harmful or adverse events were considered to be secondary to negligence and more than $90 \%$ were judged to be preventable. ${ }^{2}$ As most errors are never voluntarily reported, there is always significant failure of systems and mechanism set ups in a complex health related environment to improve patient's safety. Healthcare providers are usually extremely embarrassed about their errors or mistakes. In most cases, this issue leads to making furious attempts. They actually immediately shift the blame to someone else or something else to conceal their mistakes and in other words to defend themselves. Disclosure of errors to patients has long been fully endorsed by professional organizations and bioethicists because it respects patient autonomy, enhances informed decision making and upholds the physician decision to tell the truth .Although the majority of clinicians endorsed the principle of medical error disclosure to patients, evidence reveals that such disclosure may be uncommon. Two national surveys highlighted how huge the gap between the principle and practice is and suggests that physicians may be disclosing as few as $30 \%$ of harmful errors to the patients. ${ }^{2}$ Wrong site surgery may be the most regrettable and harmful error a surgeon can commit. It can actually lead to devastating outcomes, the consequences extend beyond the patient's imagination leading to serious legal and professional implications. Neurosurgery is the third most vulnerable specialty to wrong site surgery behind orthopedic and general surgery. ${ }^{3}$ These specialties are

Copyright (C) 2017 The Author(s). This is an open-access article distributed under the terms of the Creative Commons Attribution License (http://creativecommons.org/licenses/by/4.0), which permits unrestricted use, distribution, and reproduction in any medium, provided the original work is properly cited. 
consistent with the potential risk factors for wrong site surgery such as multiple surgeons may be involved in a case, and time pressures causing verification steps to be rushed or skipped. Wrong site craniotomy effects both patients and surgeons and will lead to definitely further exacerbating clinical outcomes. Cohen et al. ${ }^{4}$ carried out an in-depth case series on wrong-site craniotomy: analysis of 35 cases and systems for prevention. Despite the implementation of preoperative checklists and surgical time-outs, wrong-site surgery still occurs today. The authors searched medical, legal and media databases including Medline, LexisNexis, Westlaw (from 1966 to July 1,2009) and medial searches and also contacted medical licensing boards which yielded 34 cases of wrong-site craniotomy. They surveyed 50 medical licensing boards by using a brief survey instrument administered by email to the medical councils. Four categories of factors that contribute to wrong-site craniotomy were revealed after analyzing the cases. The categories are as follows: communication breakdowns, inadequate preoperative checks (technical factors and imaging) and human errors. This study is an examination and discussion of a case study in which an ethical dilemma presents the results of the wrong-site craniotomy error disclosure in a patient. This topic has been chosen as it is significant to the author, the occurrence of it is unique in neurosurgery and has caused a great deal of news and concern regarding its ethical and moral dilemma. The aim of this review is to evaluate and assess current evidence regarding the application of the Deontological theory in error disclosure of wrong-site craniotomy using a case study. Authors examined the duty of physicians and surgeons (with particular interest in neurosurgery) to disclose errors they had committed from a less paternalistic view using the moral philosophy of Immanuel Kant.

\section{Methods}

A comprehensive literature search was performed to establish evidence for an informed and evidenced based discussion. Current literature, relating to wrong-site craniotomy error disclosure or wrong site neurosurgery error disclosure were gathered by using databases including Medline and EMBASE following Preferred items for Reporting of Systematic Review and Metaanalysis (PRISMA). The keywords employed in this search strategy included deontology, wrong-site craniotomy, error disclosure, and ethical dilemma. The inclusion criteria included any type of study relevant to the review, studies on adult human patients, papers published in English, and seminal papers relevant to this study. Editorials, comments, and correspondences were excluded.

\section{Results}

In terms of the quality of the gathered evidence, only peer reviewed papers had the required quality. Generally, there is insufficiency in regards to the literature of the ethics of wrong site craniotomy. Actually, only two articles met the criteria (Cohen and $\mathrm{Wu}$ et al. ${ }^{4,5}$ ). A total of 232 articles were gathered, but after removing duplicates, editorial comments and correspondences only two articles met the criteria (Fig 1).

\section{Articles identified through database search}

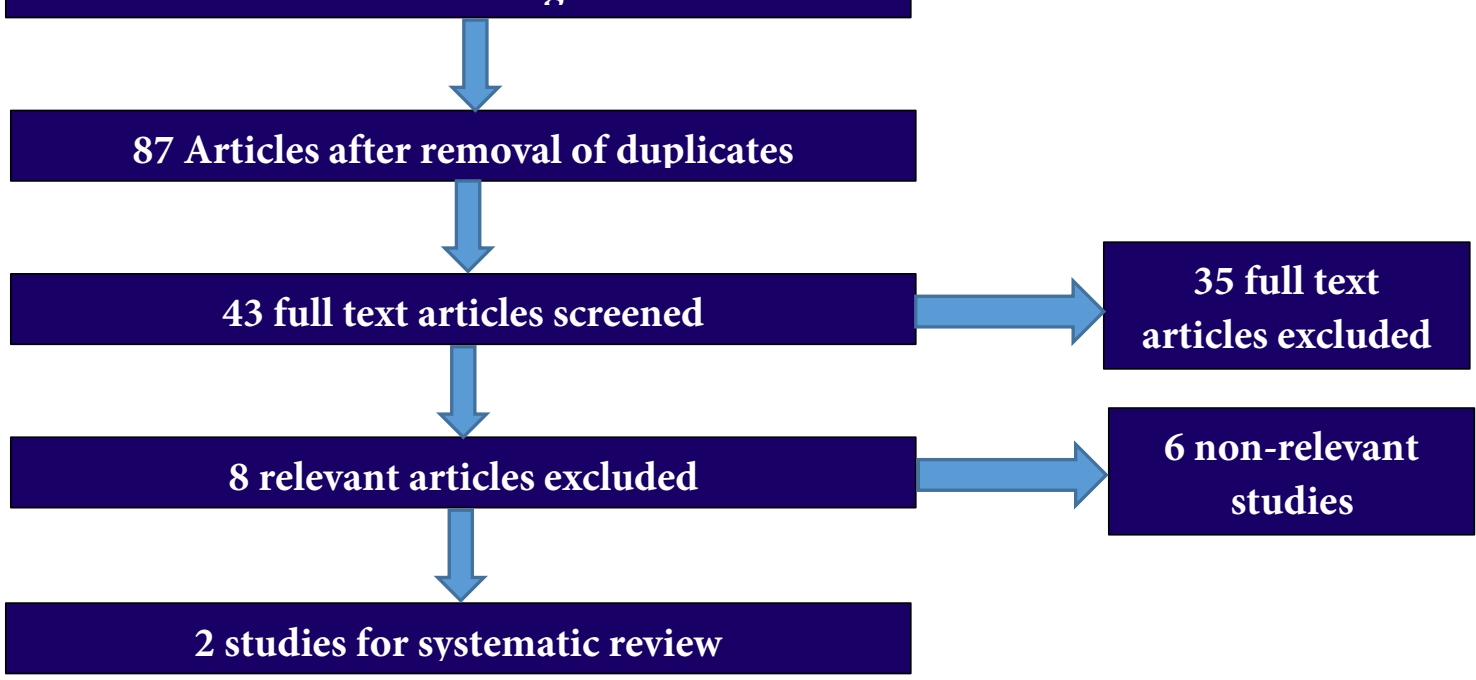

Figure 1. Search Flow Diagram

\section{Discussion}

The deontological theory implies that medical practitioners should adhere to their obligations and duties when analyzing ethical dilemma. This means that a person will perform his obligations as upholding one's duty is considered to be ethically correct. ${ }^{6}$ Furthermore, the moral obligation for surgeons to openly recognize their fallibility and attempt to develop tools and strategies to minimize medical errors, may lead to the need for disclosure. The deontological theory was at first defined by a German philosopher named Immanuel Kant who spent most of his life in Konigsberg, Prussia (1724-1804) where he studied Philosophy and Physics. 
This theory greatly contributed to the development of the deontological theory. It has been well known that this theory is one of the main theories at the foundation and origin of medical bioethics. Two main types of normative ethical system exist which are: teleological or consequence-based, and deontological or duty-based. The main teleological is the Unitarianism theory which is based on the research and writing of two British philosophers, Jeremy Bentham and John Stuart Mills. ${ }^{7}$ Also, the main deontological theory is based on what Immanuel Kant wrote and was latter translated into several volumes. ${ }^{7}$ He believes that the moral worth of an action is not at all related to the outcome it brings. It is actually related to the action being done with a sense of duty or obligation. He states "the moral worth of an action does not lie in the effect expected from it and so too does not lie in any principle of action that need to borrow its motive from this expected effect. ${ }^{8}$ What this really means is to behave not just with the sense of duty or obligation or any other motive, as duty or obligation is to actually do the right thing. In deontology, some duties are absolute. These are the obligations to do certain types of actions. Kant named this general type of obligation "categorical imperative". These actions are imperative because they fall within a certain category. Furthermore, Kant states that there is only a single categorical imperative or unconditional command governing the morality of human behavior. This command says "act only on the maxim through which you can at the same time will it to become a universal law". The Principle of the Doctrine of Virtue, is to "act on a maxim through which you can and at the same time will that it should become a universal law". ${ }^{8}$

The medicine ethos is generally built on deontology principle: no one will deny that doctors have duties. In several ways deontology represents the voice of the patient. Healthcare professionals must always keep their promises and must never lie but sometimes this is not enough today's complicated world. Wrong-site craniotomy is a huge ethical issue characterized with some level of difficulty in decision making. Medical ethics in practice is simply the application of moral principles to specific situations requiring solutions. This discussion attempts to apply the deontology theory to ascertain how far error disclosure can go and also any lessons which can be learned for future reference and reflective practice. Four major principles have been derived from the moral theories including beneficence, non-maleficence, autonomy and justice which are very popular among health care practitioners and are accepted as moral ethical codes guiding morality in decision making 9. "Beneficence" actually guides the practitioner to help his patients and leads them not to harm them (non-maleficence), "Justice" is the principle of non-discrimination. Physicians must work for the welfare of the entire society. "Autonomy" refers to the respect for patients and their rights include informed consent, professional secrecy and respect for the independence of his colleagues' professional decisions. ${ }^{10}$ There is still the question of whether medical errors should be disclosed to patients and to what extent irrespective of the outcomes, or whether they are obvious to the patients or not. In our case study of wrong-site craniotomy which is a serious and obvious error, to not disclose would mean telling a blatant and obvious lie which is unacceptable to deontology ethics. A lie is defined as merely an unintentional untrue declaration of another. It actually harms another most of the time, even if that other is not another individual, nevertheless humanity generally, inasmuch as it makes the source of right unusable. It is said that the fruitfulness has a specific duty which makes no distinction between those of whom has this duty and to whom one can exempt oneself from it. This is due to the fact that it is actually instead an unconditional duty which one holds in all relations. ${ }^{8}$ There is nothing from the Deontological theory which allows neurosurgeons to hide this type of error from a patient. If the patient asks, "did everything go well?", not disclosing the error would be considered as an act of lie, deception and preservation of narrow professional interest over the well-being of patents, which is a breach of professional ethics. On the other hand, if the patient doesn't ask, it's the surgeon's duty to disclose major errors. Physicians may tend to lie for the fear of lawsuits and other professional consequences. However, the Deontological theory insists physicians to disclose medical errors no matter what the consequences or outcomes will turn out to be.

The Kant deontology theory is not absolute and inflexible; it may allow room for times when conflicting roles arise. This theory describes two domains of obligation. When these two domains conflict with each other, the applied philosophy says that the stronger domain of obligation has priority. For example, when a physician's opinion about error disclosure in a certain condition where the patient is completely unaware of, and as a result if becoming aware of the error might do more harm than good by the anxiety and lack of evidence it evokes, the stronger ground of obligation will reside in not telling the error while the weaker ground of obligation will reside in telling the truth. Considering the ethical dilemma in wrong site craniotomy, there is a link between the decision-making process and management risk application while there may be other cognitive and psychological connections. Also, behavioral associations and verbal communication interactions occur between groups and individuals in the course of rationalization of problems. The theoretical concepts that effect and guide the process are really considered. ${ }^{11}$ The deontology theory has been significantly influential in the development of ethics to guide the moral conductions of all health careers. ${ }^{12}$ One important discussion which has been mentioned by Kant in the deontology theory is the kingdom of end. This discussion states that "Act in such a way you will always treat ... another person, never simply as a means but always at the same time as an 
end". ${ }^{8}$ What this actually means is that it is acceptable to benefit from an interaction with people as far as it's not the sole motivation of the specific interaction. Despite a global significant comprehensive generalizability and application of generic codes of professional, ethical and moral conduct, the wrong-site craniotomy still remains a controversial and abnormal cosmetic outlook condition. Hence the neurosurgical community must embrace a culture of openness and understanding towards errors to move forward and ultimately improve the quality of patient care. Wrong-site craniotomies are errors that are eradicable. ${ }^{4} \mathrm{Wu}$ et al. ${ }^{5}$ carried out a survey on disclosing medical errors to patients, which is a reflection of the deontology theory. They found out that patients will favorably respond to physicians who apologies and accept responsibility for medical errors than those who do not apologies or give ambiguous responses. Results revealed that disclosure does not seem to increase the tendency to sue among those who had been aware of an error. Nevertheless, the ethical imperative should compel a full disclosure to patients who suffered preventable harms. In this case study, the surgeon displayed full and strict deontological theory in practice, took full responsibility despite the lawsuit and negative outcome, above all settled out of court.

There are several assertions that support and refute the importance, usefulness and limitation of deontology in the consideration of ethical dilemma. Most ethicists feel that deontology is less useful in difficult situations and circumstances. For example, in triage deontology cannot tell what duties must be prioritized over others since some duties are absolute. However, the deontological theory was set up in order to establish a uniform medical practice at a behavioral level with the ability to generate the necessary skills of both doctors and patients. The professional and also human behavior of the physician who was considered to be worthy and optimal by his guild has been expressed in the medical deontological code. Discounting the outcomes as a valid factor in evaluating the morality of an action is considered to be a big criticism the of deontology ethics theory. Obviously it's not appropriate to completely ignore the outcomes, but at the same time it's not knowledgeable to rely on an outcome sole as in utilitarianism and consequentialism. Deontological theory is weaker when it comes to inform us about how to live or develop a character of virtue. Disclosure of large-scale adverse events is a challenging dilemma, not all the events were alike and this difference has implications for disclosure. Disclosure should be a

\section{References}

1. De Ville K, Elliot C. To err is human: America culture, history and medical error. In: Rubin SB, Zolot L, Editors, margin of error: The ethics of mistakes in the practice of medicine. Haggerstown: University publishing group; 2000.

2. Leape LL, Brennan TA, Laird N, Lawthers AG, Localio AR, Barnes BA, et al. The nature of adverse events in hospitalized patients. Results of the Harvard Medical Practice Study II. N Engl J Med. 1991;324(6):377-84. doi: 10.1056/NEJM1991 02073240605. pmid: 1824793. norm, even with low probability of harm. Not much detail was said about the mechanism and steps for disclosure. Although the majority of the cases had disclosure to patients and authority, some other were not. Deontological ethical error has been extremely significant in the key development of bioethical theory to guide the moral conduct of surgeons and other members of the health care profession. Deontology strongly believes that all the major accidental errors that occur when facing patients should be disclosed. This is due to the fact that surgeons are duty-bound to do the right thing including not lying, respecting patients' dignity, practicing beneficence, sympathy and acting with gratitude and conscience without arrogance. Above all, never treating a patient as a means to an end but an end in itself. Three main ways surgeons can deal with errors are acknowledge the error, apologies to the patient and family as the case may be and acquire knowledge that will help to avoid same error in the near future.

\section{Conclusions}

Evidence based approach is of paramount importance in interpretation of ethical theory to modern day multidisciplinary practice. Deontology theory can initiate meaningful debate to facilitate robust decisionmaking. Regardless of which ethical theory or combination of ethical theories a neurosurgeon decides consciously or unconsciously to employ to facilitate decision-making, the key focus should always be the patients' best interests. Some interpret that deontology may allow withholding of information from patients especially if disclosing such information will do more harm than good, but purists will not agree with this. However not all answers are on this controversial issues hence further studies on deontology of error disclosure are needed. This study is characterized with the lack of evidenced based articles on deontology and error disclosure, which is concerned to be a significant limitation of this study.

\section{Ethical Approval}

Not applicable.

\section{Authors' Contributions}

TA and AZ contributed equally to this study.

\section{Conflict of Interest Disclosures}

Author declares that he has no competing interests.

Funding

None.

3. Organizations $\mathrm{JCOAoH}$. A follow-up review of wrong site surgery 2002 [cited 2014 May 7]. Available from: http://www.jointcomission.org/SentinelEvents/SentinelEvent sAlerts/sea_24.html.

4. Cohen FL, Mendelsohn D, Bernstein M. Wrong-site craniotomy: analysis of 35 cases and systems for prevention. J Neurosurg. 2010;113(3):461-73. doi: 10.3171/2009.10.JN S091282. pmid: 20020843. 
5. Wu AW, Huang IC, Stokes S, Pronovost PJ. Disclosing medical errors to patients: it's not what you say, it's what they hear. J Gen Intern Med. 2009;24(9):1012-7. doi: 10.1007/s11606-009-1044-3. pmid: 19578819.

6. Penslar R. Research Ethics: Cases and Materials. Bloomington: Indiana University Press; 1995.

7. Bentham J. An introduction to the principles of morals and legislation (chapters $\mathrm{i}-\mathrm{v}$ ). Oxford: Clarendon press; 1970.

8. Rebbeck TR, Couch FJ, Kant J, Calzone K, DeShano M, Peng $\mathrm{Y}$, et al. Genetic heterogeneity in hereditary breast cancer: role of BRCA1 and BRCA2. Am J Hum Genet.
1996;59(3):547-53. pmid: 8751855.

9. Beauchamp T, Childress J. Principle of Biomedical Ethics. 6th ed. London: Oxford University Press; 2009.

10. Post S, Gale T. An Ethical Framework for Managed Care. Encyclopedia of Bioethics. 3rd ed. USA: Macmillan Reference; 2004.

11. Gert B, Culver CM, Clouser KD. Bioethics; A systematic approach. 2nd ed. New York: Oxford University Press; 2006.

12. Bernstein $M$, Brown B. Doctors' duty to disclose error: a deontological or Kantian ethical analysis. Can J Neurol Sci. 2004;31(2):169-74. pmid: 15198440. 